\section{Into the generation of application}

\author{
Karol Sikora
}

Hybridoma Technology in the Biosciences and Medicine. Edited by Timothy A. Springer. Plenum: 1985. Pp.602. \$75, \&71. Human Hybridomas and Monoclonal Antibodies. Edited by E.G. Engelman. S.K. Foung. J. Larrick and A. Raubitschek. Plenum: 1985. Pp.526. \$69.50, f66.

Since the discovery ten years ago of monoclonal antibodies, several books about these biologically useful tools have been produced. Until now, however, they have tended to concentrate heavily on methodology. But we are beginning to see the emergence of second generation volumes which provide the reader with much more sophisticated detail on the application of the technology. These two books are in this category.

The first, edited by Timothy Springer from Harvard University, starts with a rather dry title. However, in it the editor has managed to get together a group of authors who take us through some of the more elegant approaches to the construction and characterization of monoclonal antibodies. Recent developments in hybridoma technology involved in the production of monoclonal antibodies - peptide immunization, the expression of cloned immunoglobulin genes, and exon shuffling and class switching - are all considered and fully referenced. I know of no other source to date where this information can be found in one place. The merger of gene cloning and the monoclonal antibody fields promises to bring mutual benefit. Designer monoclonal antibodies - that is those produced with the required secondary functions by recombinant gene technology, may now become a reality, whilst the speed of cloning defined genes can be aided by having an appropriate antibody to the resulting protein.

The second section consists of a series of reviews on the biomedical application of monoclonal antibodies. Some of these are rather unoriginal and now dated, especially those concerning clinical applications. The final section considers T-lymphocyte clones and their products. Unfortunately this field has moved too fast for the publisher and although there are some useful background reviews little recent information is to be found. Altogether this is an interesting volume, containing much useful reference material about new ways to produce and improve monoclonal antibodies, but it could easily have been shortened without detriment by removing some of the reviews on specific applications.

The production of human monoclonal antibodies has captured the imagination of many over the past five years. Their
T-lymphocyte hybridomas. There follows an appendix listing methods for tissue culture and assaying supernatants and recipes for media. Lists such as these have little to do with human monoclonal antibody production specifically and are readily available elsewhere: so, again. the book is unnecessarily long despite having a useful summary of current work in this difficult area. The considerable interest of the biotechnology industry here, together with recent developments in handling and expressing human genes, will almost certainly ensure rapid development of this field.

Now that monoclonal antibodies are impinging on wide areas of biological and clinical science there is a great need for books to function as knowledge conversion kits similar to those available for upgrading construction toys for children. This would save readers considerable time and money.

Karol Sikora is the Director of the Ludwig Institute for Cancer Research. Medical Research Council Centre. Hills Road. Cambridge CB2 2QH, UK.

\section{Journey to adulthood}

\author{
Leonard A. Freed
}

The Growth and Development of Birds. By Raymond J. O'Connor. Wiley: 1984. Pp.315. £22,\$34.15.

BIRDS are extremely varied with respect to the size and composition of their eggs, the nature of the nests they provide for eggs and neonates, and growth and development both during incubation and after hatching. They range from species without parental care following egg-laying to those in which care continues to long past the fledging stage.

Growth and development occur in: (i) a thermal environment influenced by nest, parental brooding, brood size, and maturation of individual endothermy; (ii) a nutritional environment influenced by diet and frequency of feedings; (iii) a social environment in which there is parentoffspring interaction, sibling rivalry for parental attention, and interaction with conspecifics during dispersal, migration and recruitment for breeding; and (iv) a demographic environment with risks of mortality at each life-history stage. The nature and combinations of these environments differ between taxonomic groups (indicating phylogenetic constraints on developmental patterns), as well as within them for species in different ecological circumstances (indicating evolutionary adaptation of developmental patterns).

Raymond O'Connor's The Growth and Development of Birds is an important first review of aspects of these environments through its attempt to combine physiological, behavioural and ecological studies within an evolutionary perspective. The book is structured around the avian lifecycle, bringing together discussion of an unusually wide range of topics - including those mentioned above - and comparing altricial and precocial species for many of them. The goal of the book is clearly breadth rather than depth of coverage. But while specialists may be disappointed by the absence of certain details to do with their particular research interest, or the scarcity of work published after 1980, they will undoubtedly be stimulated by seeing their area put in context and perhaps moved to examine aspects of it they might previously have overlooked.

Readers should be aware that several topics (for example asynchronous hatching and optimization of growth rates) have become increasingly controversial since the book was written, and also that most examples are based on North Temperate species. Tropical birds, with different life histories and social structures, remain understudied in all aspects of growth and development. Nevertheless, the book will be read with profit by a wide range of biologists, and beginning graduate students and undergraduates will here encounter features of growth and development that they would be unlikely to come across in any single course. the Department of Zoology. University of Hawaii at Manoa, Honolulu. Hawaii 96822. USA.

- Academic Press has just published the third volume in the series Form and Function in Birds edited by A.S. King and J. McLelland; the emphasis in this volume is upon locomotion and the special sense organs. Price is $\$ 99.50$. $£ 9$ ).
Leonard A. Freed is an Assistant Professor in 